\title{
MARCKSL1 exhibits anti-angiogenic effects through suppression of VEGFR-2-dependent Akt/PDK-1/mTOR phosphorylation
}

\author{
BOH-RAM KIM ${ }^{1,4^{*}}$, SEUNG-HOON LEE ${ }^{2 *}$, MI-SUN PARK $^{1}$, SEUNG-HEE SEO ${ }^{1,4}$, \\ YOUNG-MIN PARK ${ }^{3}$, YOUNG-JOO KWON ${ }^{4}$ and SEUNG-BAE RHO ${ }^{1}$ \\ ${ }^{1}$ Research Institute, National Cancer Center, Ilsandong-gu, Goyang-si, Gyeonggi-do 410-769; \\ ${ }^{2}$ Department of Life Science, Yong In University, Cheoin-gu, Yongin-si, Gyeonggi-do 449-714; \\ ${ }^{3}$ Department of Biological Sciences, Sungkyunkwan University, Jangan-gu, Suwon-si, Gyeonggi-do 440-746; \\ ${ }^{4}$ College of Pharmacy, Graduate School of Pharmaceutical Sciences, Ewha Global Top 5 Program, \\ Ewha Womans University, Seoul 120-750, Republic of Korea
}

Received June 30, 2015; Accepted August 10, 2015

DOI: $10.3892 / o r .2015 .4408$

\begin{abstract}
Myristoylated alanine-rich C kinase substratelike 1 (MARCKSL1) plays a pivotal role in the regulation of apoptosis and has been shown to maintain antitumor and metastasis-suppressive properties. In the present study, we examined the effects of MARCKSL1 as a novel anti-angiogenic agent on the inhibition of angiogenesis-mediated cell migration. MARCKSL1 also reduced vascular endothelial growth factor (VEGF)-induced human umbilical vein endothelial cell (HUVEC) proliferation, as well as capillary-like tubular structure formation in vitro. MARCKSL1 disrupted phosphorylation of vascular endothelial growth factor receptor-2 (VEGFR-2) in ovarian tumorigenesis. In addition, MARCKSL1 showed potent anti-angiogenic activity and reduced the levels of VEGF and hypoxia-inducible factor $1 \alpha$ (HIF-1 $\alpha$ ) expression, an essential regulator of angiogenesis. Consistently, MARCKSL1 decreased VEGF-induced phosphorylation of the PI3K/Akt signaling pathway components, including phosphoinositide-dependent protein kinase 1 (PDK-1), mammalian target of rapamycin (mTOR), tuberous
\end{abstract}

Correspondence to: Dr Seung Bae Rho, Research Institute, National Cancer Center, 323 Ilsan-ro, Ilsandong-gu, Goyang-si, Gyeonggi-do 410-769, Republic of Korea

E-mail: sbrho@ncc.re.kr

*Contributed equally

Abbreviations: MARCKSL1, myristoylated alanine-rich C kinase substrate-like 1; PKC, protein kinase C; MRP, MARCKS-related protein; VEGFR-2, vascular endothelial growth factor receptor 2; HUVECs, human umbilical vein endothelial cells; PDK-1, phosphoinositide-dependent protein kinase 1; mTOR, mammalian target of rapamycin; TSC-2, tuberous sclerosis complex 2; p70S6K, p70 ribosomal protein S6 kinase; GSK-3 $\beta$, glycogen synthase kinase $3 \beta$

Key words: MARCKSL1, anti-angiogenic activity, protein-protein interaction, Akt/PDK-1/mTOR phosphorylation, endothelial cells sclerosis complex 2 (TSC-2), p70 ribosomal protein S6 kinase (p70S6K), and glycogen synthase kinase $3 \beta$ (GSK-3 $\beta$ ) protein. Collectively, our results provide evidence for the physiological/ biological function of an endothelial cell system involved in angiogenesis through suppression of Akt/PDK-1/mTOR phosphorylation by interaction with VEGFR-2.

\section{Introduction}

Members of the myristoylated alanine-rich $\mathrm{C}$ kinase substrate (MARCKS) family, as specific protein kinase C (PKC) substrates, modulate the PKC signaling pathway via its phosphorylation. PKC has been implicated in cell proliferation, and recent studies indicate that MARCKS may function as a cell growth suppressor. For example, MARCKS and MARCKSrelated protein (MRP) are essential proteins implicated in various cellular events such as cell motility, adhesion, secretion and phagocytosis in many cell types $(1,2)$. Myristoylated alanine-rich C kinase substrate-like 1 (MARCKSL1) is part of the MARCKS family and an essential cellular substrate for PKC as a membrane-bound protein. MARCKSL1 (also known as MacMARCKS, MLP, MRP or F52) has been associated with the coordination of membrane-cytoskeletal signaling events such as regulation of integrin activation, cell adhesion, cell spreading, cell migration and brain development, as well as phagocytosis, mitogenesis, membrane trafficking, endocytosis and exocytosis. It is expressed in a variety of tissues with the highest levels found in the testis and uterus (3-8). Li et al (9) reported that MARCKS is a target of miR-21, a microRNA that promotes tumor invasion and metastasis in a number of human cancers, suggesting that it is an important tumor suppressor. Recent research suggests that c-Jun N-terminal kinase (JNK) phosphorylation of MARCKSL1 regulates actin homeostasis, formation of lamellipodium and filopodium, and neuronal migration under physiological conditions in neurons and when ectopically expressed in prostate cancer cells (8). Kim et al (10) reported that MARCKSL1 provides a novel molecular mechanism for regulating cancer metastasis and can serve as a therapeutic target for LOXL2-associated diseases, including acting as a tumor suppressor. 
Angiogenesis is a critical process in endothelial cell development, movement, reproduction and degradation of the extracellular matrix, as well as in wound repair. The complex multi-step procedure of angiogenesis plays an important role in cell division and metastasis in the majority of solid tumors. In addition, angiogenic stimulators of endothelial cell functions are associated with new blood vessel formation during embryonic development, such as vascular endothelial growth factor (VEGF), which plays a major role in the proliferation, migration and invasion of vascular endothelial cells (11-13). For example, VEGF plays a pivotal role in the progression of ovarian and colon cancers by controlling cancer growth through enhanced tumor angiogenesis. Recent studies have emphasized the crucial role of the Wnt signaling pathway for embryo/organ-specific endothelial cell differentiation in vasculogenesis (14-19). In addition, VEGF upregulation has been associated with various diseases. Solid tumors cannot grow beyond a certain size without an adequate blood supply; however, tumors expressing VEGF are able to grow and metastasize. VEGF acts via two specific binding receptor tyrosine kinases, namely, VEGFR-1/flt-1 and VEGFR-2/KDR/flk-1, both of which are expressed on normal vascular endothelial cells (20). VEGFR-2 plays an important role in the activation of essential downstream regulators responsible for endothelial cell proliferation including migration, invasion, survival and embryonic angiogenesis (21-23), while VEGFR-1 is not associated with the proliferation of endothelial cells (24). The VEGF/VEGFR-2 signaling pathway is required for maintenance of vascular homeostasis in various tissues and organs $(25,26)$.

Physiological endothelial cell activity plays an important role in regulating various vascular-related mechanisms and vascular-associated diseases, including tumor growth and maintenance. The detailed biological function of MARCKSL1 in endothelial cells during ovarian tumor growth remains unclear. Generally, the role of angiogenesis is examined based on cell proliferation, migration and capillary-like tubule formation in endothelial cells. In the present study, we investigated the direct effect of MARCKSL1 on anti-angiogenic activity in tumor angiogenesis using an in vitro human umbilical vein endothelial cell (HUVEC) system. We also identified the molecular regulators responsible for VEGFR-2/Akt/mammalian target of rapamycin (mTOR)-dependent endothelial cell growth during tumorigenesis. MARCKSL1 inhibited phosphorylation of signaling modulators downstream of phosphatidylinositol3'-kinase (PI3K) such as Akt, phosphoinositide-dependent protein kinase 1 (PDK-1), mTOR and tuberous sclerosis complex 2 (TSC-2) via direct interactions with VEGFR-2. These interactions control powerful anti-angiogenic activities. Therefore, our results strongly suggest that MARCKSL1 plays a major role in regulating cellular angiogenesis.

\section{Materials and methods}

Cell culture and antibodies. Human ovarian cancer cell line OVCAR-3 was seeded in Dulbecco's modified Eagle's medium (DMEM) (Life Technologies, Gaithersburg, MD, USA) supplemented with $10 \%$ heat-inactivated fetal bovine serum (FBS), penicillin $(100 \mathrm{U} / \mathrm{ml}) /$ streptomycin $(100 \mu \mathrm{g} / \mathrm{ml})$. Primary HUVECs (Clonetics, Walkersville, MD, USA) were cultured on $0.3 \%$ gelatin-coated dishes (Sigma, St. Louis, MO, USA) using
EGM-2 BulletKit medium (Clonetics). All cells were maintained in a humidified atmosphere containing $5 \% \mathrm{CO}_{2}$ at $37^{\circ} \mathrm{C}$. The following primary antibodies were used in the present study: anti-mTOR, anti-phospho-mTOR, anti-p70S6K, antiphospho-p70S6K, anti-GSK-3 $\beta$, anti-phospho-GSK-3 $\beta$ (Cell Signaling, Beverly, MA, USA), anti-MARCKSL1, anti-Flag, anti-VEGFR-2, anti-phospho-VEGFR-2, anti-HIF-1 $\alpha$, anti-PI3K, anti-phospho-PI3K, anti-Akt, anti-phosphoAkt, anti-PDK-1, anti-phospho-PDK-1, anti-TSC-2 and anti-phospho-TSC-2 (Santa Cruz Biotechnology, Santa Cruz, CA, USA), anti-VEGF ${ }_{121}$ (Ab-1; Oncogene, Cambridge, MA, USA) and $\beta$-actin (Sigma).

Migration and capillary tube formation assay. Endothelial cell migration analyses were performed according to a previously described protocol using Transwell chambers $(8-\mu \mathrm{m}$ pore size; Costar) $(27,28)$. Briefly, the lower surface of the filter was coated with $10 \mu \mathrm{g}$ gelatin. M199 containing $1 \%$ FBS with VEGF (10 ng/ml) was placed in the lower wells. After $24 \mathrm{~h}$, the cells were fixed with $99 \%$ methanol and stained with hematoxylin and eosin (H\&E). Cells were estimated as previously reported $(27,28)$. For the in vitro tube formation assays (27,28), $200 \mu 110 \mathrm{mg} / \mathrm{ml}$ growth factor-reduced Matrigel was added into a 24 -well plate and polymerized at $37^{\circ} \mathrm{C}$ for $30 \mathrm{~min}$. Non-transfected (control/empty-inserted vector only), MARCKSL1 or MARCKSL1 siRNA (siMARCKSL1)-transfected HUVECs $\left(2.5 \times 10^{5}\right)$ were seeded on the surface of the Matrigel. Seeded whole cells were then incubated for $48 \mathrm{~h}$ with or without $10 \mathrm{ng} / \mathrm{ml}$ VEGF in M199 containing 1\% FBS. Tubular structure formation lengths were measured using an inverted microscope equipped with a digital CCD camera (Zeiss), and quantification was estimated with ImageLab imaging software (MCM Design).

$\left[{ }^{3} H\right]$ thymidine incorporation assay. To calculate cell proliferation, HUVECs were seeded at a density of $1.9 \times 10^{4} /$ well in standard medium in gelatinized plates on day 0 . Next, the $\left[\right.$ methyl $\left.-{ }^{3} \mathrm{H}\right]$ thymidine incorporation rate was evaluated according to a previously described protocol using a liquid scintillation counter (Beckman Instrument) $(27,28)$.

Immunoblot analysis. For whole protein extraction, the cultured cells were harvested, washed with phosphatebuffered saline (PBS), centrifuged and disrupted by adding ice-cold lysis buffer containing protease inhibitors $(50 \mathrm{mM}$ Tris, $\mathrm{pH} 7.2,150 \mathrm{mM} \mathrm{NaCl}, 1 \%$ Triton $\mathrm{X}-100,1 \mu \mathrm{g} / \mathrm{ml}$ leupeptin, $1 \mu \mathrm{g} / \mathrm{ml}$ pepstatin, $2 \mu \mathrm{g} / \mathrm{ml}$ aprotinin and $200 \mu \mathrm{g} /$ $\mathrm{ml}$ phenylmethylsulfonyl fluoride) for $1 \mathrm{~h}$ at $4^{\circ} \mathrm{C}$. Proteins were subsequently separated by $8-12 \%$ SDS-PAGE and transferred to Immobilon-P membranes (Millipore Corporation, Billerica, MA, USA). After blocking, the membranes were incubated with the indicated primary antibodies at $4^{\circ} \mathrm{C}$ overnight. The membranes were washed three times in TBST washing buffer and incubated with horseradish peroxidase-conjugated secondary antibodies. Protein bands were detected using the ECL detection system (GE Healthcare, Little Chalfont, Buckinghamshire, UK).

Yeast two-hybrid $(\mathrm{Y} 2 \mathrm{H})$ assay and quantitation of interaction. For bait construction with human MARCKSL1,cDNA encoding 
full-length human MARCKSL1 was subcloned into the EcoRI and $X h o$ I restriction enzyme sites of the pGilda/LexA yeast shuttle cloning vector. The bait pGilda/LexA-MARCKSL1 plasmid was introduced into a yeast strain EGY48 [MATa, his3, trp1, ura3-52, leu2::pLeu2-LexAop6/pSH18-34 (LexAop-lacZ reporter)] using a modified lithium acetate method $(29,30)$. Human VEGFR-1 and VEGFR-2 were introduced, with each cDNA encoding a full-length gene into the multi-cloning restriction enzyme sites of the pJG4-5 plasmid vector, which included B42 fusion proteins (Clontech, Palo Alto, CA, USA). The VEGFR-1 and VEGFR-2 cDNAs encoding pJG4-5 fusion proteins were transformed into competent yeast cells that already contained pGilda/LexA-MARCKSL1, while the transformants were selected based on their tryptophan prototrophy (plasmid marker) on a synthetic medium (Ura, His, Trp) containing $2 \%(\mathrm{w} / \mathrm{v})$ glucose. The binding activity of the interaction was estimated according to a previously described protocol (30).

Co-immunoprecipitation assay. For co-immunoprecipitation (co-IP), the cells were trypsinized and then centrifuged. Cell pellets were rinsed with ice-cold PBS and re-suspended in RIPA lysis buffer (50 mM Tris/HCl, pH 7.2, $150 \mathrm{mM} \mathrm{NaCl}$, $1 \%$ Triton $\mathrm{X}-100$, protease inhibitor cocktail containing $1 \mu \mathrm{g} / \mathrm{ml}$ leupeptin, $1 \mu \mathrm{g} / \mathrm{ml}$ pepstatin, $2 \mu \mathrm{g} / \mathrm{ml}$ aprotinin and $200 \mu \mathrm{g} / \mathrm{ml}$ PMSF). The cell lysates were then incubated with anti-Flag antibody (Santa Cruz) and precipitated with protein A-agarose (GE Healthcare). Approximately $20 \mu \mathrm{g}$ of the precipitated proteins was separated by $10-12 \%$ SDS-PAGE and transferred to Immobilon-P membranes. After blocking, the membranes were incubated with the indicated primary antibodies, including anti-MARCKSL1 and anti-VEGFR-2. Immunoreactive bands were visualized using the ECL detection system (GE Healthcare).

Luciferase reporter-gene assay. In vitro VEGFR-2 promoter assays were performed as previously described (31). Briefly, cells at $85 \%$ confluency were transfected with a VEGFR-2 reporter expression vector. After lysis in ice-cold RIPA buffer, whole cell lysates were cleared by centrifugation for $15 \mathrm{~min}$ at $14,000 \mathrm{rpm}$, and cell extracts were incubated at room temperature with the luciferase substrate reagent for $30 \mathrm{~min}$ according to the manufacturer's instructions. Then, a 5- $\mu$ l aliquot of each sample was examined using a MicroLumat Plus LB96V luminometer.

Statistical analysis. All data are presented as means \pm SD and were assessed using the Student's t-test. Significant differences of $95 \%$ confidence $(\mathrm{P}<0.05)$ are depicted by an asterisk $\left(^{*}\right)$ on each graph.

\section{Results}

MARCKSL1 inhibits VEGF-induced endothelial cell migration, capillary tube formation and cell proliferation in vitro. During the multi-step process of angiogenesis, one of the crucial stimulators during early development is VEGF, which induces cell migration, invasion, proliferation and differentiation of endothelial cells. Endothelial cell activity plays an important role in controlling various vascular-associated functions and diseases, including tumor cell growth and maintenance. To determine whether MARCKSL1 controls the effects of VEGF on cell migration in HUVECs, angiogenesis was examined based on cell migration, capillary-like tubular structure formation and cell proliferation in endothelial cells. The inhibitory effects of MARCKSL1 on VEGF-induced migration of endothelial cells were first assessed using Transwell migration assays. As expected, VEGF promoted the migration of the non-transfected cells and MARCKSL1-control (empty-inserted vector)-transfected cells compared with non-stimulated cells. Our results indicated that MARCKSL1 overexpression noticeably disrupted VEGF-induced cell migration, while MARCKSL1-siRNA did not (Fig. 1A). We performed further experiments using various concentrations of MARCKSL1 or siMARCKSL1 based on transient transfection. As shown in Fig. 1B, VEGF prompted the cell migration of the empty MARCKSL1-transfected HUVECs compared with the unstimulated cells, as expected. However, overexpression of MARCKSL1 gradually inhibited VEGF-induced cell migration in a dose-dependent manner. Inhibition of MARCKSL1 expression via MARCKSL1-siRNA maintained the stimulatory effects of VEGF on HUVEC migration. Therefore, overexpression of MARCKSL1 potently inhibits key events in the angiogenic process induced by VEGF, such as the migration of endothelial cells in vitro. Subsequently, we investigated the anti-angiogenic effects of MARCKSL1 on the formation of VEGF-induced capillary-like tubular structures on Matrigel using an in vitro angiogenesis model system in HUVECs. As shown in Fig. 1C, untreated or control cells incubated with VEGF formed a capillary-like structure on Matrigel. In contrast, the overexpression of MARCKSL1 completely inhibited VEGF-induced tubular structure formation. The inhibitory effect of MARCKSL1 on VEGFinduced capillary tube formation was completely recovered by siMARCKSL1 transient transfection. Finally, the inhibitory effects on VEGF-induced proliferation of endothelial cells were assessed using a $\left[{ }^{3} \mathrm{H}\right]$ thymidine incorporation assay system. Generally, VEGF enhanced DNA synthesis of the untransfected HUVECs, and the control (empty-inserted vector)-transfected HUVECs were comparable to the noninduced cells (32). MARCKSL1 significantly suppressed VEGF-induced DNA synthesis in HUVECs (Fig. 1D). Therefore, overexpression of MARCKSL1 suppresses key events in VEGF-induced angiogenesis in vitro, including endothelial cell migration and cell proliferation. Collectively, these findings indicate that MARCKSL1 specifically controls VEGF-induced cell migration and tube formation, as well as the proliferation of HUVECs.

MARCKSL1 tumor suppressor protein inhibits VEGF-induced VEGFR-2 phosphorylation via the interaction with VEGFR-2 protein. VEGFR-2 is an essential receptor involved in angiogenesis and vasculogenesis and controls endothelial cell migration, proliferation and mitogenesis. VEGFR-2 has been implicated primarily in cancer metastasis and tumorigenesis. To address the possible mechanism behind the inhibition of angiogenesis by the tumor suppressor MARCKSL1, we used the $\mathrm{Y} 2 \mathrm{H}$ protein-protein interaction assay and the co-IP analysis system. We first investigated the inhibitory effect of MARCKSL1 on VEGF-induced VEGFR-2 protein expression 


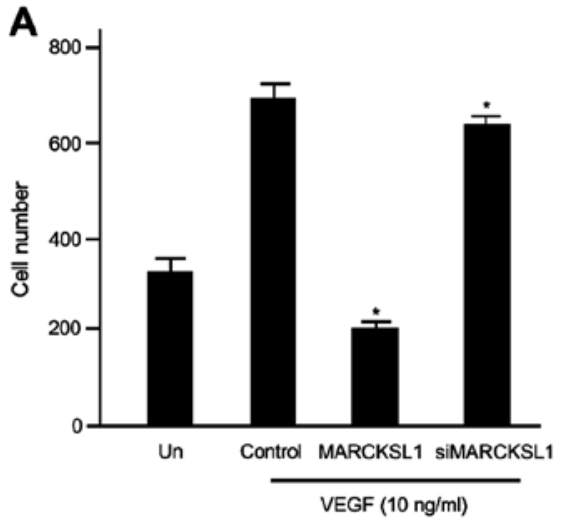

B
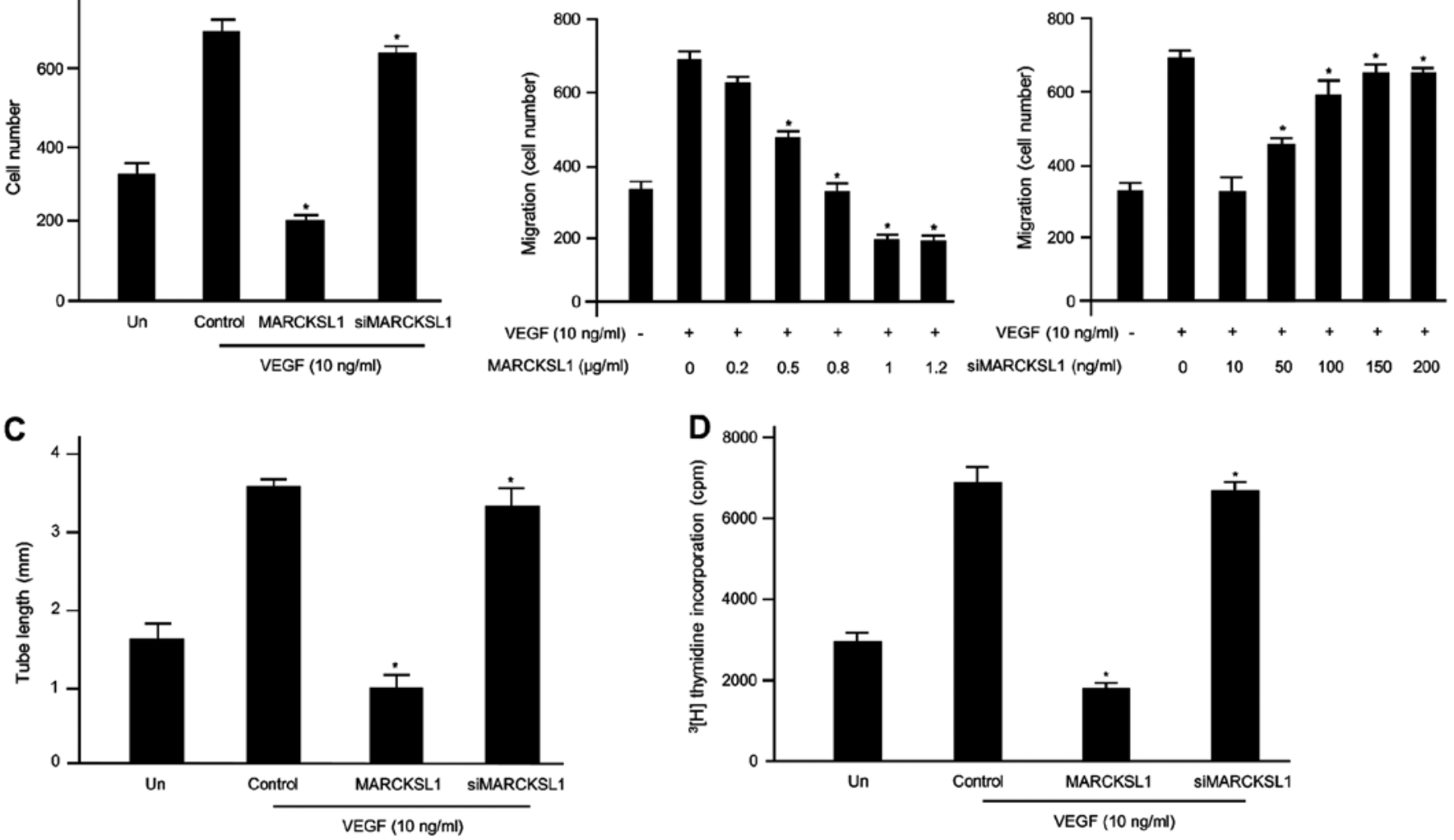

Figure 1. MARCKSL1 inhibits VEGF-induced endothelial cell migration, tube formation and cell proliferation in vitro. (A) The anti-angiogenic effect of human MARCKSL1 on HUVEC migration. Transfectants were assessed using Matrigel-coated Transwells for the migration assay system, followed by induction for $48 \mathrm{~h}$ in the absence or presence of VEGF. The number of cells that migrated was measured under a light microscope. Each bar represents the mean \pm SD of three independent experiments that yielded similar results. ${ }^{*} \mathrm{P}<0.05$. (B) After treatment with VEGF, HUVECs were transfected with MARCKSL1 (left panel) or siMARCKSL1 (right panel) constructs at various concentrations. All cells were fixed and then stained with H\&E. The number of migrated cells was estimated under a light microscope and is indicated as the mean $\pm \mathrm{SD}$. ${ }^{*} \mathrm{P}<0.05$ vs. control group. (C) Effect of human MARCKSL1 and siMARCKSL1 on HUVEC tubular structure formation. Each transfectant was maintained on growth factor-reduced Matrigel and then treated for $48 \mathrm{~h}$ with or without VEGF. Quantification of the newly formed tubule networks was performed based on images captured from an inverted microscope. Data values are represented as the mean \pm SD from three independent experiments. Significant differences of $95 \%$ confidence ("P $<0.05)$ are depicted for each graph. (D) The inhibitory effect of MARCKSL1 on endothelial cell proliferation. Cells were seeded for 3 days with or without VEGF. The cpm value of [ $\left.{ }^{3} \mathrm{H}\right]$ thymidine was estimated using a liquid scintillation counter. The data represent the mean $\pm \mathrm{SD}$ of three independent experiments. ${ }^{*} \mathrm{P}<0.05$ compared with the control group.

in ovarian carcinoma cells. Overexpression of MARCKSL1 significantly reduced the phosphorylation of VEGFR-2, whereas siMARCKSL1 completely reversed the inhibitory effect (Fig. 2A). VEGF rapidly activates angiogenesis in tumor metastasis as well as tumorigenesis; hence, we evaluated the inhibitory effect of MARCKSL1 on VEGF protein expression in OVCAR-3 tumor cells. The overexpression of MARCKSL1 completely reduced VEGF expression, whereas siMARCKSL1 reversed the inhibitory effect (Fig. 2B). We also demonstrated the effect of MARCKSL1 on hypoxia-inducible factor $1 \alpha$ $(H I F-1 \alpha)$ protein expression. As a transcription factor, HIF-1 $\alpha$ is an important molecule for VEGF protein expression. As shown in Fig. 2B, MARCKSL1 markedly decreased the expression of the HIF-1 $\alpha$ protein. The inhibitory effect of MARCKSL1 was completely recovered by siMARCKSL1 transient transfection. These observations indicate that VEGF-induced protein expression is notably inhibited by the MARCKSL1 tumor suppressor. Direct interactions among various proteins are critical for the majority of cellular functions. For example, signaling cascades from the exterior to the interior of a cell are regulated through protein-protein interactions. Thus, signal transduction processes play a pivotal role in cellular/physiological/biological processes, as well as in aggressive solid tumors and various diseases. Therefore, protein-protein interactions are important for the majority of processes in a living cell microenvironment. Thus, we investigated the cellular interaction between VEGFR-2 and MARCKSL1 protein in an in vivo and in vitro system. VEGFR-1 and the expression vector only (empty-inserted vector) were used as negative controls. As shown in Fig. 2C, the $\beta$-galactosidase activity between MARCKSL1 and VEGFR-2 was fully activated $(86.88 \pm 1.12)$, which was not the case with the expression vector only (empty-inserted vector) $(1.61 \pm 0.57)$ or VEGFR-1 $(1.97 \pm 0.56)$. To further confirm this direct interaction between MARCKSL1 and VEGFR-2 observed in the $\mathrm{Y} 2 \mathrm{H}$ assay system, we explored this relationship using co-IP analysis. Bait constructs of MARCKSL1 (pcDNA3.1/MARCKSL1) and VEGFR-2 (pcDNA3.1/Flag-VEGFR-2) or pcDNA3.1/Flag-VEGFR-2 and expression plasmid vector only (pcDNA3.1) were co-transfected into HEK293T cells. Continuously, IP was performed using anti-Flag primary antibody with whole cell 
A

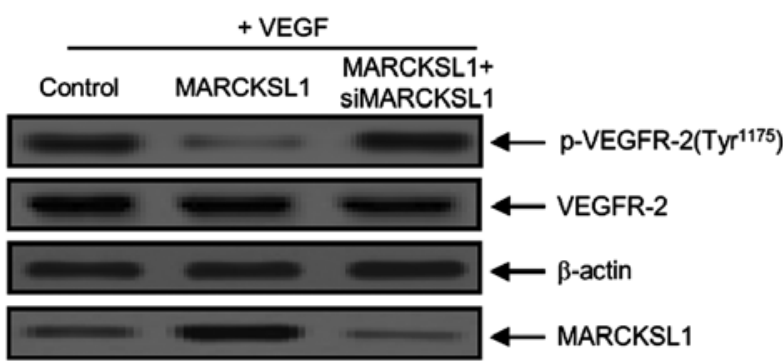

C

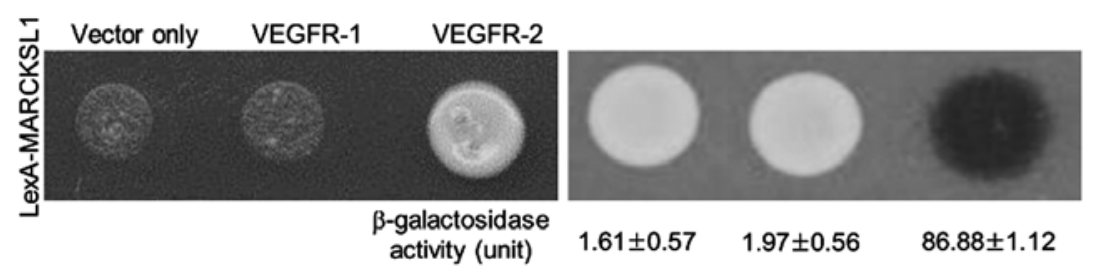

B

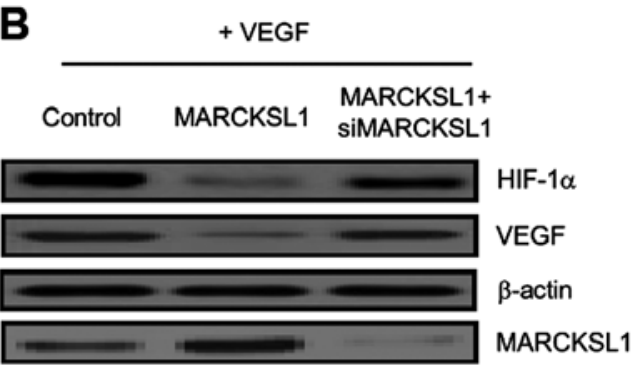

E

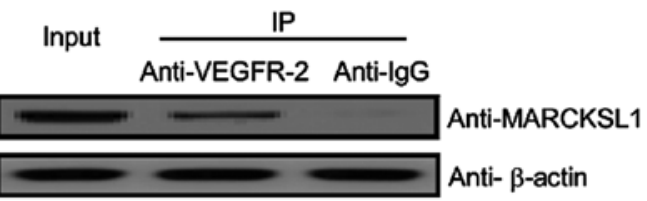

$\mathbf{F}$

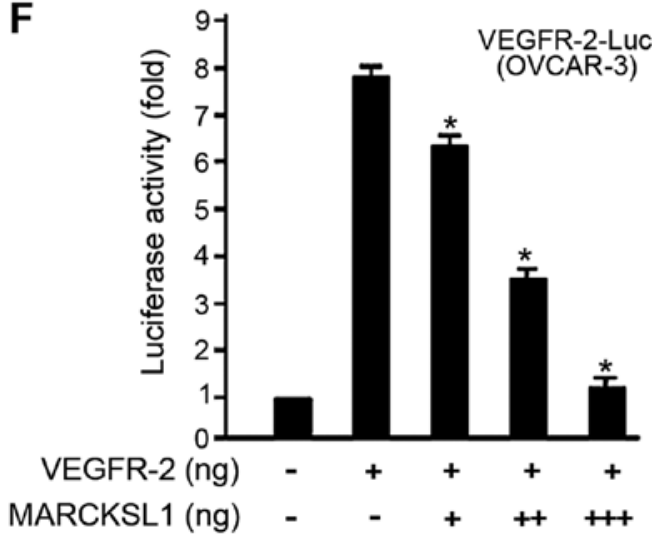

D

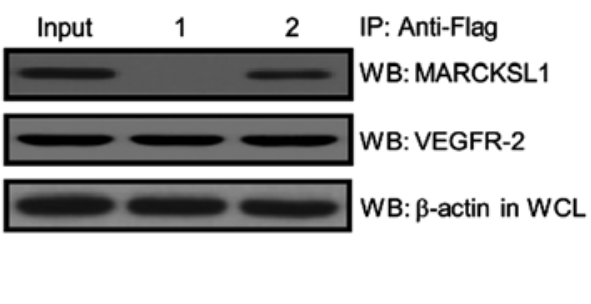

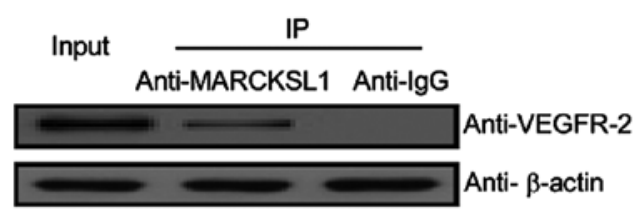

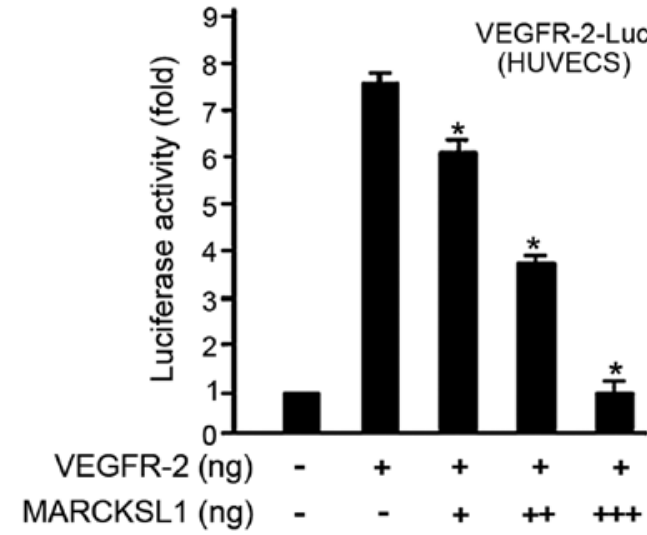

Figure 2. MARCKSL1 can bind to VEGFR-2, but not VEGFR-1. (A) Cells were treated with VEGF and then transfected with either control (empty-inserted vector only) or MARCKSL1 or MARCKSL1 plus siMARCKSL1. Tumor suppressor MARCKSL1 effectively reduced the phosphorylation (Tyr1175) of VEGFR-2 triggered by VEGF. Phosphorylation of VEGFR-2 was detected using a specific primary antibody. Non-phosphorylated VEGFR-2 and $\beta$-actin were used to verify equal sample loading. (B) Cells were incubated with $10 \mathrm{ng} / \mathrm{ml}$ VEGF and then transfected with either control (empty-inserted vector) or MARCKSL1 or MARCKSL1 plus siMARCKSL1. HIF-1 $\alpha$ and VEGF expressions were then detected using western blot analysis. Three independent experiments were performed in triplicate. (C) Positive-protein interactions were monitored as cell growth on plates lacking leucine (left panel) and by the formation of black colonies on X-gal plates containing galactose. The values of $\beta$-galactosidase activity (unit) estimated by adding O-nitrophenyl $\beta$-D-galactopyranoside (ONPG) are indicated below the corresponding lanes (right panel). (D) Co-immunoprecipitation of MARCKSL1 with VEGFR-2. Immunoprecipitation was performed using an anti-Flag-specific primary antibody with whole lysates from both transfected HEK293T cells. After immunoprecipitation, total proteins $(20 \mu \mathrm{g})$ were immunoblotted using anti-MARCKSL1 and anti-VEGFR-2 primary antibodies. Lane 1, pcDNA3.1 (expression plasmid vector only) and pcDNA3.1/Flag-VEGFR-2 transfectant; lane 2, pcDNA3.1/MARCKSL1 and pcDNA3.1/Flag-VEGFR-2 transfectant. (E) Co-immunoprecipitation of endogenous MARCKSL1 with VEGFR-2 shows the interaction of the two proteins. All experiments were performed at least three times with similar results. (F) Inhibition of VEGFR-2-dependent transcriptional activity by MARCKSL1. OVCAR-3 carcinoma cells (left panel) and HUVECs (light panel) were co-transfected with $500 \mathrm{ng}$ VEGFR-2-Luc, 500 ng VEGFR-2-inserted expression plasmid vector (pcDNA3.1/VEGFR-2), and increasing concentrations of the gene encoding MARCKSL1 (pcDNA3.1/Flag-MARCKSL1) $(100,250$ and $500 \mathrm{ng}$ ). The results shown are representative of at least three independent experiments. The values are expressed as means \pm SD. Significant differences of $95 \%$ confidence ( $\mathrm{P}<0.05)$ are depicted for each graph. " $\mathrm{P}<0.05$ compared with the control group.

lysates from both transfected cells. After IP, the precipitated proteins were detected using either anti-MARCKSL1 or anti-VEGFR-2 primary antibody. As shown in Fig. 2D, VEGFR-2 co-immunoprecipitated with MARCKSL1 (lane 2 in the upper panel). Next, we demonstrated the interaction between endogenous MARCKSL1 and VEGFR-2. As shown in Fig. 2E, endogenous MARCKSL1 directly co-immunoprecipitated with VEGFR-2. These results were 
A
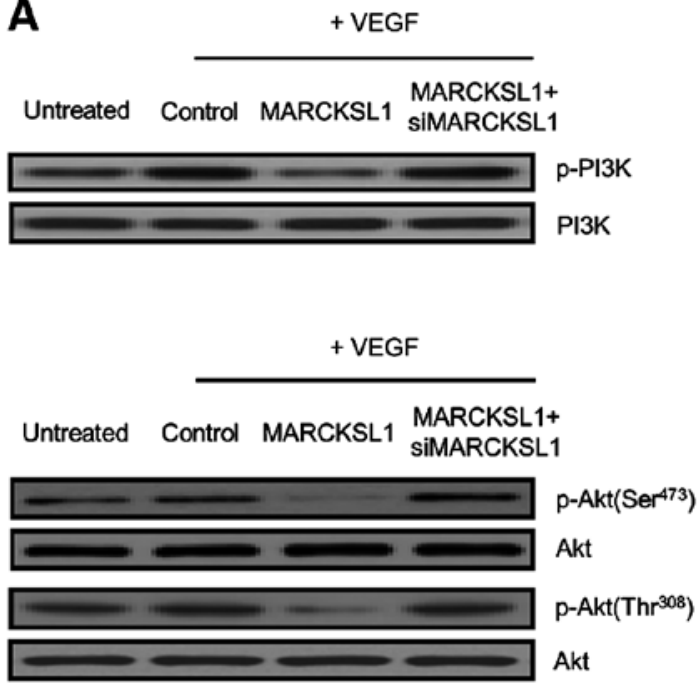

B

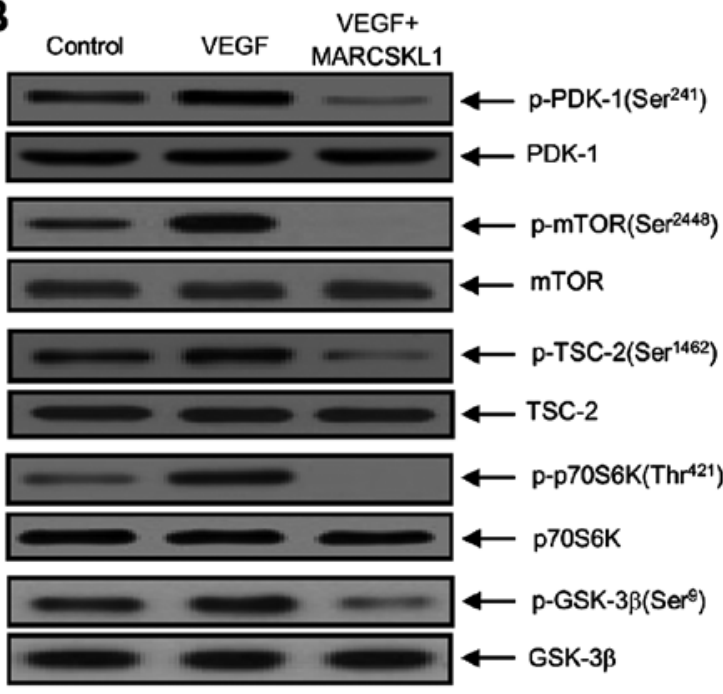

Figure 3. Disrupted phosphorylation of downstream components in the VEGFR-2/Akt/mTOR signaling pathways by the MARCKSL1 tumor suppressor. (A) Phospho-PI3K and phospho-Akt suppression by MARCKSL1 disclosure in OVCAR-3 ovarian tumor cells. Cells were incubated with 10 ng/ml VEGF and transfected with either the control (empty-inserted vector only), MARCKSL1 or MARCKSL1 plus siMARCKSL1. Phosphorylation of PI3K and Akt (Ser473 and Thr308) were then developed by western blot analysis. Non-phosphorylated PI3K and Akt were used as the loading control. Three independent experiments were performed in triplicate. Protein levels were measured based on densitometric analysis and normalized to levels of the loading control (data not shown). (B) The phosphorylation effects of MARCKSL1 on PDK-1 (Ser241), mTOR (Ser2448), TSC-2 (Ser1462), p70S6K (Thr421) and GSK-3 3 (Ser9) signaling components, such as in the cases of upstream and downstream target regulators of Akt. After transfection with MARCKSL1, equal amounts of cellular total protein $(20 \mu \mathrm{g})$ were separated by SDS-PAGE, followed by immunoblotting with indicated primary antibodies specific to the phosphorylated proteins (p-PDK-1, p-mTOR, p-TSC-2, p-p70S6K and p-GSK-3 $\beta$ ). Immunoblotting of non-phosphorylated PDK-1, mTOR, TSC-2, p70S6K and GSK-3 $\beta$ were used to verify equal sample loading. All experiments were performed at least three times with similar results.

indicative of the binding between endogenous VEGFR-2 and MARCKSL1 in the cells. Finally, to determine whether MARCKSL1 regulates VEGFR-2, the effect of MARCKSL1 on VEGFR-2 transcription activity was estimated based on a luciferase reporter gene assay using a construct fusing the VEGFR-2 promoter to the luciferase gene. Luciferase activity was gradually reduced by transient transfection of MARCKSL1 in a dose-dependent manner (Fig. 2F), further supporting the importance of MARCKSL1 for the regulation of VEGFR-2 activity. Our results strongly indicate that overexpression of MARCKSL1 reduces its transcriptional activity. Collectively, the results demonstrated that MARCKSL1 disrupts the VEGFR-2 transcript levels by inhibiting VEGFR-2 phosphorylation via the Akt/mTOR signaling pathway.

Disrupted phosphorylation of downstream components in the VEGFR-2/Akt/mTOR signaling pathways by the MARCKSL1 tumor suppressor. PI3K/Akt phosphorylation is a crucial step in the regulation of fundamental cellular processes involved in tumor angiogenesis and tumor growth. Akt, as a pivotal downstream regulator of $\mathrm{PI} 3 \mathrm{~K}$, induces mTOR through a variety of cellular functions, including phosphorylation and inactivation of apoptotic cell death-associated proteins (33-35). Therefore, to address the detailed functional mechanism underlying its effects, we examined whether MARCKSL1 reduced PI3K and Akt phosphorylation in OVCAR-3 tumor cells. As indicated in Fig. 3A, VEGF-induced PI3K and Akt phosphorylation were significantly reduced by the MARCKSL1 tumor suppressor. The inhibitory effect of MARCKSL1 on VEGF-induced phosphorylation was nearly recovered by transient transfection of siMARCKSL1. These results suggested that MARCKSL1 specifically suppresses VEGF-stimulated
PI3K/Akt phosphorylation in ovarian tumor cells. We also investigated the phosphorylation effects on upstream and downstream signaling components of the PI3K/Akt pathway that control tumor endothelial cell function in the inhibition of angiogenesis. For example, p70 ribosomal protein S6 kinase (p70S6K), a mitogen-activated serine/threonine kinase, is an essential regulator of protein synthesis and plays an important role in cell growth, proliferation, differentiation and survival. As indicated in Fig. 3B, MARCKSL1 reduced VEGF-induced phosphorylation of the PI3K/Akt signaling cascade components, including PDK-1, TSC-2, mTOR, p70S6K and glycogen synthase kinase-3 $\beta$ (GSK-3 $\beta$ ). Taken together, these results suggest that MARCKSL1 has a novel biological function in activating apoptosis, as well as inhibiting angiogenesis via concomitant inactivation of VEGF-stimulated phosphorylation of the cascade components in the VEGFR-2/Akt/mTOR signaling pathways.

\section{Discussion}

Previous research showed that LOXL2 recovers MARCKSL1induced apoptosis through inhibition of the FAK/Akt/mTOR signaling pathway in carcinoma cells (10). According to this study, increased LOXL2 expression activated cellular metastasis by suppressing MARCKSL1-induced apoptotic cell death by regulating cell cycle- and apoptosis-associated protein expression, including p21, cyclin D1, p53 and Bcl-2 family genes. Second, LOXL2 was found to directly bind to MARCKSL1, and MARCKSL1 overexpression reduced LOXL2 expression in carcinoma cells. Third, induction of the LOXL2-mediated FAK/Akt/mTOR signaling pathways and tumor metastasis were decreased by overexpression 
of MARCKSL1. In the present study, we validated that MARCKSL1 had an inhibitory effect on VEGF-induced angiogenesis in the endothelial cell system.

Angiogenesis plays a pivotal role in both early development and progression of ovarian cancer, as well as in normal ovarian function $(36,37)$. As demonstrated via histologic studies, ovarian tumors are highly neovascularized and microvascular number and pathological/biological aggressiveness are interrelated $(38,39)$. As in other malignant tumors, angiogenesis is an essential factor for ovarian carcinoma growth. VEGF is associated with accelerated angiogenesis during the early stages of ovarian carcinoma; thus, VEGF-induced angiogenesis is a vital early regulator in ovarian carcinogenesis $(36,40)$. Generally, angiogenic signaling cascades are mediated by VEGF and their receptors, VEGFRs (41). VEGF expression is closely associated with the regulation of tumor metastasis, growth, migration, invasion and aggression, as well as poor survival (42-44). Notably, most human solid tumors show upregulated expression of VEGF and HIF-1 $\alpha$, which enhance tumor angiogenesis and tumor growth. Thus, suppression of tumor angiogenesis by disturbed VEGF and HIF-1 $\alpha$ expression is becoming a pivotal approach for carcinoma treatment $(45,46)$. One study reported that maximal inhibition of tumor growth and tumor angiogenesis can be accomplished by disrupting VEGF circulation (47). Herein, we demonstrated a novel cellular function for MARCKSL1 as a novel potent angiogenic modulator that can disrupt VEGF and HIF-1 $\alpha$ expression in the VEGFR-2/Akt/mTOR signaling pathway using a cancer model system. As indicated in Fig. 1, overexpression of MARCKSL1 considerably reduced the major events of VEGF-induced angiogenesis in vitro, involving endothelial cell migration and cell proliferation. At the same time, overexpression of MARCKSL1 completely abrogated the VEGF-induced capillary-like tubular structure network. In the presence of MARCKSL1-siRNA, endothelial cells were not affected. Taken together, these results indicate that MARCKSL1 specifically regulates VEGF-induced HUVEC migration and tube formation. HIF-1 $\alpha$ enhances the transcript levels of many genes such as VEGF, transferrin and endothelin-1 in tumor metastasis and neovascularization (48-50). In addition, the HIF-1 $\alpha$ protein is commonly upregulated in various types of human tumors and in regional and distant metastases (51). Therefore, its activation is closely associated with gene expression, tumor angiogenesis, and tumor growth (52). Fang et al (53) reported that apigenin significantly reduces the expression of VEGF and HIF-1 $\alpha$ in ovarian tumor cells. As mentioned above, HIF-1 $\alpha$, as a transcription factor, is an essential mediator of VEGF protein expression. As indicated in Fig. 2B, MARCKSL1 markedly decreased the expression of VEGF and HIF-1 $\alpha$ protein. In contrast, the inhibitory effect of MARCKSL1 was completely restored by siMARCKSL transfection. VEGFR-2 is phosphorylated through its interaction with VEGF, and its downstream signaling cascade activates PI3K/Akt phosphorylation, which is a potent cytokine in the control of endothelial cell growth and survival of various carcinomas, including ovarian tumors (46,54-57). Akt phosphorylates mTOR and is regulated by mTOR through a negative and positive feedback system (35), and it also acts as a major modulator of cell proliferation, promoting cell survival through various cellular mechanisms. Despite these studies, the cellular mechanisms of ovarian tumor angiogenesis remain unclear. Therefore, to address the detailed functional mechanism underlying its effects, we investigated whether MARCKSL1 downregulated PI3K and Akt phosphorylation in OVCAR-3 tumor cells. Overexpression of MARCKSL1 notably inhibited phosphorylation of VEGFR-2, whereas siMARCKSL1 completely reversed the inhibitory effect (Fig. 2A). In addition, VEGF-induced PI3K and Akt phosphorylation were considerably reduced by the MARCKSL1 tumor suppressor. The inhibitory effect of MARCKSL1 on VEGF-induced phosphorylation was nearly recovered by MARCKSL1-siRNA transient transfection (Fig. 3A). Consistently, MARCKSL1 decreased VEGF-induced phosphorylation of the PI3K/Akt signaling cascade components, including PDK-1, TSC-2, mTOR, p70S6K and GSK-3 $\beta$. Collectively, these results suggest that MARCKSL1 disturbs VEGFR-2 transcriptional activity by restraining VEGFR-2 phosphorylation via the Akt/PDK-1/mTOR signaling pathway.

In summary, the present study examined the anti-angiogenic effects of the tumor suppressor MARCKSL1 in ovarian tumorigenesis using an in vitro model system. In the present study, we further revealed a novel biological/physiological function and cellular molecular mechanism for MARCKSL1, which serves as an anti-angiogenic factor that can mediate phosphorylation of Akt/PDK-1/mTOR through inhibition of VEGF and HIF-1 $\alpha$ expression levels via interaction with VEGFR-2. Therefore, combination therapy with MARCKSL1 and traditional antitumor reagents may be a useful approach for more advanced ovarian cancer, recurrent and certain other cancer patients.

\section{Acknowledgements}

This study was supported through a grant from the National Cancer Center, Korea (NCC-1510050-1 and 1410312-2).

\section{References}

1. Larsson C: Protein kinase $\mathrm{C}$ and the regulation of the actin cytoskeleton. Cell Signal 18: 276-284, 2006.

2. Kalwa $\mathrm{H}$ and Michel T: The MARCKS protein plays a critical role in phosphatidylinositol 4,5-bisphosphate metabolism and directed cell movement in vascular endothelial cells. J Biol Chem 286: 2320-2330, 2011.

3. Aderem A: The MARCKS brothers: A family of protein kinase $C$ substrates. Cell 71: 713-716, 1992.

4. McNamara RK and Lenox RH: Distribution of the protein kinase $\mathrm{C}$ substrates MARCKS and MRP in the postnatal developing rat brain. J Comp Neurol 397: 337-356, 1998.

5. Underhill DM, Chen J, Allen LA and Aderem A: MacMARCKS is not essential for phagocytosis in macrophages. J Biol Chem 273: 33619-33623, 1998.

6. Yue L, Lu S, Garces J, Jin T and Li J: Protein kinase C-regulated dynamitin-macrophage-enriched myristoylated alanine-rice $C$ kinase substrate interaction is involved in macrophage cell spreading. J Biol Chem 275: 23948-23956, 2000.

7. Arbuzova A, Schmitz AA and Vergères G: Cross-talk unfolded: MARCKS proteins. Biochem J 362: 1-12, 2002.

8. Björkblom B, Padzik A, Mohammad H, Westerlund N, Komulainen E, Hollos P, Parviainen L, Papageorgiou AC, Iljin K, Kallioniemi O, et al: c-Jun N-terminal kinase phosphorylation of MARCKSL1 determines actin stability and migration in neurons and in cancer cells. Mol Cell Biol 32: 3513-3526, 2012.

9. Li T, Li D, Sha J, Sun P and Huang Y: MicroRNA-21 directly targets MARCKS and promotes apoptosis resistance and invasion in prostate cancer cells. Biochem Biophys Res Commun 383: 280-285, 2009 
10. Kim BR, Dong SM, Seo SH, Lee JH, Lee JM, Lee SH and Rho SB: Lysyl oxidase-like 2 (LOXL2) controls tumor-associated cell proliferation through the interaction with MARCKSL1. Cell Signal 26: 1765-1773, 2014

11. Folkman J and Shing Y: Angiogenesis. J Biol Chem 267: 10931-10934, 1992.

12. Risau W: Mechanisms of angiogenesis. Nature 386: 671-674, 1997.

13. Ferrara N: VEGF and the quest for tumour angiogenesis factors. Nat Rev Cancer 2: 795-803, 2002.

14. van Gijn ME, Daemen MJ, Smits JF and Blankesteijn WM: The wnt-frizzled cascade in cardiovascular disease. Cardiovasc Res 55: 16-24, 2002.

15. Cohen ED, Tian Y and Morrisey EE: Wnt signaling: An essential regulator of cardiovascular differentiation, morphogenesis and progenitor self-renewal. Development 135: 789-798, 2008.

16. Zerlin M, Julius MA and Kitajewski J: Wnt/Frizzled signaling in angiogenesis. Angiogenesis 11: 63-69, 2008.

17. Dejana E, Tournier-Lasserve $\mathrm{E}$ and Weinstein BM: The control of vascular integrity by endothelial cell junctions: Molecular basis and pathological implications. Dev Cell 16: 209-221, 2009.

18. Franco CA, Liebner S and Gerhardt H: Vascular morphogenesis: A Wnt for every vessel? Curr Opin Genet Dev 19: 476-483, 2009.

19. Dejana E: The role of wnt signaling in physiological and pathological angiogenesis. Circ Res 107: 943-952, 2010.

20. Mustonen T and Alitalo K: Endothelial receptor tyrosine kinases involved in angiogenesis. J Cell Biol 129: 895-898, 1995.

21. Breier G: Endothelial receptor tyrosine kinases involved in blood vessel development and tumor angiogenesis. Adv Exp Med Biol 476: 57-66, 2000.

22. Ferrara N: Role of vascular endothelial growth factor in regulation of physiological angiogenesis. Am J Physiol Cell Physiol 280: C1358-C1366, 2001

23. Meyer RD and Rahimi N: Comparative structure-function analysis of VEGFR-1 and VEGFR-2: What have we learned from chimeric systems? Ann NY Acad Sci 995: 200-207, 2003

24. Meyer RD, Singh A, Majnoun F, Latz C, Lashkari K and Rahimi N: Substitution of C-terminus of VEGFR-2 with VEGFR-1 promotes VEGFR-1 activation and endothelial cell proliferation. Oncogene 23: 5523-5531, 2004.

25. Yang Y, Zhang Y, Cao Z, Ji H, Yang X, Iwamoto H, Wahlberg E, Länne T, Sun B and Cao Y: Anti-VEGF-and anti-VEGF receptorinduced vascular alteration in mouse healthy tissues. Proc Natl Acad Sci USA 110: 12018-12023, 2013.

26. Cao Y: VEGF-targeted cancer therapeutics-paradoxical effects in endocrine organs. Nat Rev Endocrinol 10: 530-539, 2014.

27. Lee OH, Kim YM, Lee YM, Moon EJ, Lee DJ, Kim JH, Kim KW and Kwon YG: Sphingosine 1-phosphate induces angiogenesis: Its angiogenic action and signaling mechanism in human umbilical vein endothelial cells. Biochem Biophys Res Commun 264: 743-750, 1999.

28. Lee JH, Chun T, Park SY and Rho SB: Interferon regulatory factor-1 (IRF-1) regulates VEGF-induced angiogenesis in HUVECs. Biochim Biophys Acta 1783: 1654-1662, 2008.

29. Rho SB, Lee KH, Kim JW, Shiba K, Jo YJ and Kim S: Interaction between human tRNA synthetases involves repeated sequence elements. Proc Natl Acad Sci USA 93: 10128-10133, 1996.

30. Rho SB, Kim MJ, Lee JS, Seol W, Motegi H, Kim S and Shiba K: Genetic dissection of protein-protein interactions in multi-tRNA synthetase complex. Proc Natl Acad Sci USA 96: 4488-4493, 1999

31. Rho SB, Song YJ, Lim MC, Lee SH, Kim BR and Park SY: Programmed cell death 6(PDCD6) inhibits angiogenesis through $\mathrm{PI} 3 \mathrm{~K} / \mathrm{mTOR} / \mathrm{p} 70 \mathrm{~S} 6 \mathrm{~K}$ pathway by interacting of VEGFR-2. Cell Signal 24: 131-139, 2012.

32. Plate KH, Breier G, Weich HA and Risau W: Vascular endothelial growth factor is a potential tumour angiogenesis factor in human gliomas in vivo. Nature 359: 845-848, 1992

33. Downward J: Signal transduction. A target for PI(3) kinase. Nature 376: 553-554, 1995.

34. Khwaja A: Akt is more than just a Bad kinase. Nature 401: 33-34, 1999.

35. Guertin DA and Sabatini DM: An expanding role for mTOR in cancer. Trends Mol Med 11: 353-361, 2005.

36. Ramakrishnan S, Subramanian IV, Yokoyama Y and Geller M: Angiogenesis in normal and neoplastic ovaries. Angiogenesis 8 : 169-182, 2005.

37. Kumaran GC, Jayson GC and Clamp AR: Antiangiogenic drugs in ovarian cancer. Br J Cancer 100: 1-7, 2009.
38. Alvarez AA, Krigman HR, Whitaker RS, Dodge RK and Rodriguez GC: The prognostic significance of angiogenesis in epithelial ovarian carcinoma. Clin Cancer Res 5: 587-591, 1999.

39. Hazelton D, Nicosia RF and Nicosia SV: Vascular endothelial growth factor levels in ovarian cyst fluid correlate with malignancy. Clin Cancer Res 5: 823-829, 1999.

40. Ko YB, Kim BR, Yoon K, Choi EK, Seo SH, Lee Y, Lee MA, Yang JB, Park MS and Rho SB: WIF1 can effectively co-regulate pro-apoptotic activity through the combination with DKK1. Cell Signal 26: 2562-2572, 2014

41. Ferrara N: Role of vascular endothelial growth factor in physiologic and pathologic angiogenesis: Therapeutic implications. Semin Oncol 29 (Suppl 16): S10-S14, 2002.

42. Mu J, Abe Y, Tsutsui T, Yamamoto N, Tai XG, Niwa O, Tsujimura T, Sato B, Terano H, Fujiwara $\mathrm{H}$, et al: Inhibition of growth and metastasis of ovarian carcinoma by administering a drug capable of interfering with vascular endothelial growth factor activity. Jpn J Cancer Res 87: 963-971, 1996.

43. Hartenbach EM, Olson TA, Goswitz JJ, Mohanraj D, Twiggs LB, Carson LF and Ramakrishnan S: Vascular endothelial growth factor (VEGF) expression and survival in human epithelial ovarian carcinomas. Cancer Lett 121: 169-175, 1997.

44. Yamamoto S, Konishi I, Mandai M, Kuroda H, Komatsu T, Nanbu K, Sakahara H and Mori T: Expression of vascular endothelial growth factor (VEGF) in epithelial ovarian neoplasms: Correlation with clinicopathology and patient survival, and analysis of serum VEGF levels. Br J Cancer 76: 1221-1227, 1997.

45. Ferrara $\mathrm{N}$ and Davis-Smyth T: The biology of vascular endothelial growth factor. Endocr Rev 18: 4-25, 1997.

46. Park ST, Kim BR, Park SH, Lee JH, Lee EJ, Lee SH and Rho SB Suppression of VEGF expression through interruption of the HIF-1 $\alpha$ and Akt signaling cascade modulates the anti-angiogenic activity of DAPK in ovarian carcinoma cells. Oncol Rep 31: 1021-1029, 2014.

47. Gerber HP, Kowalski J, Sherman D, Eberhard DA and Ferrara N: Complete inhibition of rhabdomyosarcoma xenograft growth and neovascularization requires blockade of both tumor and host vascular endothelial growth factor. Cancer Res 60: 6253-6258, 2000.

48. Kerbel RS: New targets, drugs, and approaches for the treatment of cancer: An overview. Cancer Metastasis Rev 17: 145-147, 1998.

49. Semenza GL: Regulation of mammalian $\mathrm{O}_{2}$ homeostasis by hypoxia-inducible factor 1. Annu Rev Cell Dev Biol 15: 551-578, 1999.

50. Semenza GL: Hypoxia, clonal selection, and the role of HIF-1 in tumor progression. Crit Rev Biochem Mol Biol 35: 71-103, 2000.

51. Zhong H, De Marzo AM, Laughner E, Lim M, Hilton DA Zagzag D, Buechler P, Isaacs WB, Semenza GL and Simons JW: Overexpression of hypoxia-inducible factor 1alpha in common human cancers and their metastases. Cancer Res 59: 5830-5835, 1999.

52. Maxwell PH, Dachs GU, Gleadle JM, Nicholls LG, Harris AL, Stratford IJ, Hankinson O, Pugh CW and Ratcliffe PJ: Hypoxiainducible factor-1 modulates gene expression in solid tumors and influences both angiogenesis and tumor growth. Proc Natl Acad Sci USA 94: 8104-8109, 1997.

53. Fang J, Xia C, Cao Z, Zheng JZ, Reed E and Jiang BH: Apigenin inhibits VEGF and HIF-1 expression via PI3K/AKT/p70S6K1 and HDM2/p53 pathways. FASEB J 19: 342-353, 2005.

54. Altomare DA, Wang HQ, Skele KL, De Rienzo A, KleinSzanto AJ, Godwin AK and Testa JR: AKT and mTOR phosphorylation is frequently detected in ovarian cancer and can be targeted to disrupt ovarian tumor cell growth. Oncogene 23: 5853-5857, 2004

55. Olsson AK, Dimberg A, Kreuger J and Claesson-Welsh L: VEGF receptor signalling - in control of vascular function. Nat Rev Mol Cell Biol 7: 359-371, 2006.

56. Engelman JA: Targeting PI3K signalling in cancer: Opportunities, challenges and limitations. Nat Rev Cancer 9: 550-562, 2009.

57. Hanrahan AJ, Schultz N, Westfal ML, Sakr RA, Giri DD, Scarperi S, Janakiraman M, Olvera N, Stevens EV, She QB, et al: Genomic complexity and AKT dependence in serous ovarian cancer. Cancer Discov 2: 56-67, 2012. 